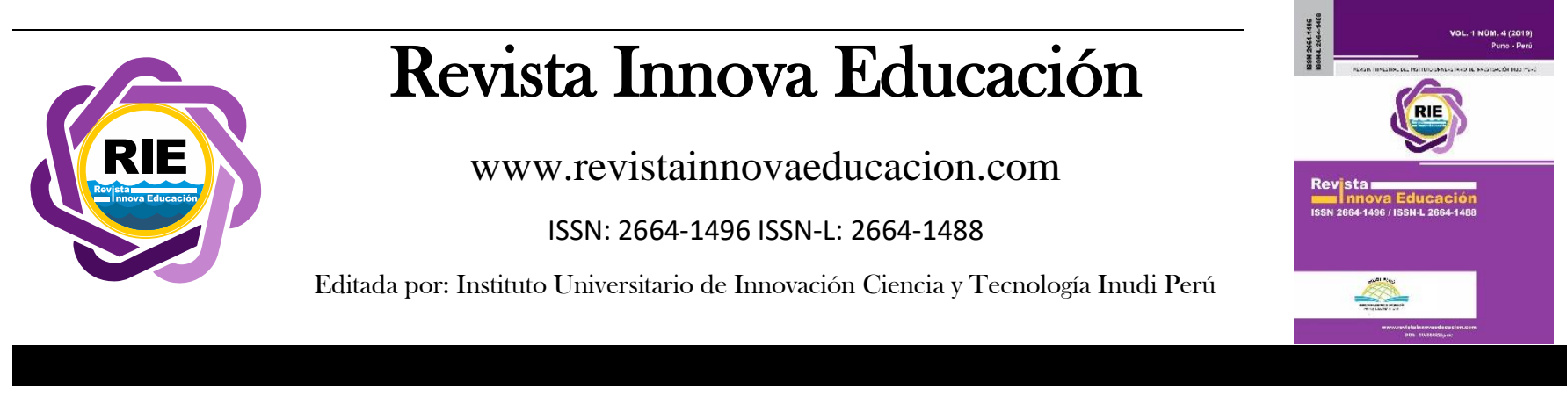

\title{
Inteligencia emocional y cultura organizacional como pilares de ejercicio docente
}

\author{
Emotional intelligence and organizational culture as pillars of teaching practice
}

\author{
Mariela Cuno \\ Universidad Nacional del Altiplano, Perú \\ (iD https://orcid.org/0000-0002-2055-5922
}

\author{
Duverly Incacutipa \\ Universidad Nacional del Altiplano, Perú \\ Dhttps://orcid.org/0000-0002-0067-3147
}

DOI: https://doi.org/10.35622/j.rie.2019.04.013

Recibido 23/03/2019/ Aceptado 30/10/219

\begin{tabular}{l} 
ARTíCULO ORIGINAL \\
\multicolumn{1}{|c|}{ PALABRAS CLAVE } \\
Liderazgo, \\
motivación, \\
organización \\
educativa, \\
institucional.
\end{tabular}

$\quad$ KEYWORDS
Leadership,
motivation,
educational
organization,
institutional climate.

RESUMEN. La investigación tuvo como propósito determinar la correlación entre inteligencia emocional y la cultura organizacional de los docentes en la Institución Educativa Andrés Avelino Cáceres de Puno, 2015 (Perú). Fue realizado desde el enfoque cuantitativo, de tipo descriptivo y de diseño correlacional transversal. La muestra estuvo constituida por 22 docentes con muestreo de carácter censal. La técnica para la obtención de datos fue la encuesta y como instrumentos se aplicaron dos cuestionarios pertinentes a cada variable. La presentación de los resultados fue a través de tablas de frecuencia, llegando a la conclusión de que existe relación significativa y positiva entre la inteligencia emocional y la cultura organizacional.

\section{INTRODUCCIÓN}

Las inteligencias múltiples es un vasto escenario para las investigaciones actuales, que nos permite explorar más allá del concepto de coeficiente intelectual. La inteligencia emocional, considerada por Goleman (1995) como la meta-habilidad que determina el grado de destreza que podemos conseguir en el dominio de nuestras otras facultades (Buey, 2002), como correlato, las personas que utilizan este tipo de inteligencia, están dotadas de una notable capacidad para comprometerse

ABSTRACT. The purpose of the research was to determine the correlation between
emotional intelligence and the organizational culture of teachers at the Institución
Educativa Secundaria Andrés Avalino Cáceres de Puno - Perú (2015). It was carried
out from the quantitative approach, descriptive type, and cross-correlational design.
The sample consisted of 22 teachers with a census sample. The technique for obtaining
data was the survey and two pertinent questionnaires were applied as instruments to
each variable. The presentation of the results was through frequency tables, reaching
the conclusion that there is a significant and positive relationship between emotional
intelligence and organizational culture.
intelligence and organizational culture. 
con las causas de otras personas, que adoptan responsabilidades, mantienen la ética y son afables en las relaciones humanas (Goleman, 1995), lo cual, es el primer eje desarrollado.

Por otro lado, como complemento idóneo, se aborda la cultura organizacional que alude al conjunto de valores, necesidades expectativas, creencias, políticas y normas aceptadas y practicadas (Schein, 1985), los cuales quedan imbricadas en un entramado de significados que rige la conducta de los seres humanos y por ende, el de la organización (Geertz, 2003).

Esta simbiosis conceptual y pragmática, en un escenario de organizaciones educativas, constituye posibilidades muy favorables para la formación de educandos preparados para los tiempos actuales, así sustentan autores que consideran que el compañerismo, la transparencia y la honestidad (Ansa, 2009), son temas tan importantes como el desarrollo de los contenidos curriculares. Considerar los objetivos organizacionales como como parte de la actividad docente (Momenti, 2015), además del compromiso y sentido de pertenencia (Hernández et al., 2018), que los maestros estén premunidos de capacidad de respuesta frente a las situaciones de crisis (Blanco, 2017), en el escenario educativo, familiar y social, pues no se trata de considerar a la escuela como una isla, sino, la continuidad de la familia.

Por lo que estas dos propuestas resultan muy relevantes como planteamiento para una nueva pedagogía que responda al diagnóstico de la educación actual de muchas instituciones, principalmente públicas, que en pleno siglo XXI, asumen aún patrones de una escuela tradicional, que ha dominado la mayor parte de las instituciones educativas a lo largo de la historia humana y en la mayoría de regiones del mundo (Diaz, 2017), priorizando una educación de corte vertical, de transmisión de conocimientos y con poco espacio para la crítica y la autocrítica, que propicie escenarios más convergentes y dialogantes en la formación de ciudadanos comprometidos en tarea de construir sociedades más solidarias.

En suma, el propósito fue determinar la relación existente entre la inteligencia emocional y la cultura organizacional de los docentes en la Institución Educativa Privada Andrés Avelino Cáceres Puno durante el periodo 2015.

\section{MÉTODO Y MATERIALES}

\section{Objetivos}

Determinar la relación entre inteligencia emocional y la cultura organizacional de los docentes en la Institución Educativa Privada Andrés Avelino Cáceres Puno, 2015.

\section{Método}

En el presente artículo se utilizó el método hipotético deductivo, de enfoque cuantitativo, orientado a describir las variables y el diseño de investigación correlacional, buscando determinar el grado de relación que existe entre la inteligencia emocional y la cultura organizacional.

\section{Población y muestra}

La población estuvo conformada por el total de docentes de la Institución Educativa 
“Andrés Avelino Cáceres” de la ciudad Puno (Perú), que hacen un total de 22 docentes del nivel inicial, primario, docentes de talleres y auxiliares. El tamaño de la muestra de la investigación estuvo conformado por el total de la población, denominándolo muestreo de carácter censal, en donde todos y cada uno de los docentes en estudio tienen la misma posibilidad de ser diagnosticados.

\section{Variables}

Las variables analizadas para medir el grado de significancia son: a) la inteligencia emocional y b) la cultura organizacional.

\section{Instrumentos}

Para el registro de datos se utilizó la encuesta y se emplearon dos instrumentos de medición. Para medir la inteligencia emocional se aplicó el Test de Inteligencia Emocional de Weisinger (1998) y para medir la cultura organizacional se aplicó el cuestionario de Olmos y Socha (2006). Ambos cuestionarios estuvieron establecidos en la escala de Likert ( $1=$ nunca y $5=$ siempre), a su vez, el coeficiente de correlación de ambos instrumentos fue de 0.721 y 0.82 , respectivamente.

\section{Diseño estadístico}

Se trabajó usando el coeficiente de correlación de Spearman, puesto que se trataron de datos cualitativos.

\section{RESULTADOS}

Los resultados expresados mediante tablas de frecuencia utilizando el programa Startical Product and Service Solutions SPSS 17.0.2, de acuerdo con la recolección de datos. Los resultados presentan dimensiones, de la misma forma, las tablas se presentan tal y como se evaluó durante la ejecución de la investigación; todo ello para dar respuesta a la interrogante: ¿Qué relación existe entre inteligencia emocional y la cultura organizacional de los docentes en la Institución Educativa Andrés Avelino Cáceres de la ciudad Puno? La relevancia de la investigación es que aborda lo que durante mucho tiempo se ha olvidado la dimensión afectiva y emocional en la educación y ésta se ha centrado en enseñar contenidos dirigidos al desarrollo cognitivo e intelectual del niño (Valenzuela \& Portillo, 2018), lo cual resulta un proceso no tan sencillo, ya que debe validar viejos esquemas para adaptar elementos nuevos, de eso se trata la educación emocional ofrece una innovación pedagógica que pretende dar respuesta a las necesidades que quedan desatendidas en la educación formal (Cebriá, 2017).

Tabla 1. Resultados Test de inteligencia emocional de los docentes de la Institución Educativa Privada Andrés Avelino Cáceres Puno.

\begin{tabular}{lllll}
\hline NIVEL & $\mathbf{f}_{\mathbf{i}}$ & $\mathbf{F}$ & \% & PH\% \\
\hline Nivel alto & 16 & 16 & 72.73 & 72.73 \\
Nivel medio & 5 & 21 & 22.73 & 95.46 \\
Nivel bajo & 1 & 22 & 4.55 & 100.00 \\
TOTAL & 22 & & 100.00 & \\
\hline
\end{tabular}

Fuente: Elaborado en función a las encuestas aplicadas. 
Se analiza el desarrollo de la inteligencia emocional en los docentes encuestados, con el nivel alto a 16 docentes que representa el $72.73 \%$ ellos muestran emociones afectivas favorables, esto significa que los docentes manejan con destreza las competencias emocionales como son: autoconciencia, autocontrol, motivación, empatía por lo que entienden que la inteligencia emocional no es sólo ser conscientes de nuestras emociones, sino asumir que éstas a su vez actúan sobre nuestros pensamientos y nuestro modo de procesar la información de manera funcional (Extremera \& Fernández, 2003) y la práctica de las habilidades sociales, es decir consideran que siempre escuchan abiertamente a sus compañeros de trabajo, manteniendo buenas relaciones sociales y laborables con sus compañeros, esto significa que la manera de relacionarse entre compañeros es buena y el ambiente de trabajo es agradable.

Mientras que el $22.73 \%$ tiene el nivel medio de inteligencia emocional, es decir, no conocen los objetivos institucionales, que el ambiente de trabajo se torna desagradable en las áreas administrativas, tampoco reconocen sus sentimientos y no responden por sus acciones laborales y personales sostienen que las relaciones sociales entre compañeros no son buenas y que existe poco trabajo en equipo.

De esta manera, la cultura no es algo neutral o estática en la organización, sino, un fenómeno dinamizador que orienta el norte de la institución, como se expresa en la tabla siguiente.

Tabla 2. Resultados de la cultura organizacional de los docentes de la Institución Educativa Andrés Avelino Cáceres, 2015

\begin{tabular}{|c|c|c|c|c|}
\hline $\begin{array}{l}\text { OPCIÓN } \\
\text { RESPUESTA }\end{array}$ & $\begin{array}{ll}D E & f_{i}\end{array}$ & $\mathbf{F}$ & $\%$ & $\mathbf{P h}_{\mathrm{h}} \%$ \\
\hline Siempre & 1 & 1 & 4.55 & 4.55 \\
\hline Frecuentemente & 16 & 17 & 72.73 & 77.28 \\
\hline A veces & 5 & 22 & 22.73 & 100.00 \\
\hline Casi nunca & 0 & 0 & 0 & \\
\hline Nunca & 0 & 0 & 0 & \\
\hline TOTAL & 22 & & 100.00 & \\
\hline
\end{tabular}

Fuente: Elaborado en función a la aplicación de encuestas.

Se observa que el $77.28 \%$ muestran que la organización cultural es favorable en la IE Andrés Avelino Cáceres, mostrando actitudes positivas en el modo de vida, un sistema de creencias y valores con un solo objetivo, una forma aceptada de interacción y relaciones típicas de organización, por tanto presencian un sistema de significados compartidos entre los docentes y que distingue a una de las otras, que se forman a lo largo de la vida de la organización como resultado de las interacciones entre sus miembros, de éstos con las estructuras, estrategias, sistemas, procesos, y de la organización con su entorno, a partir de las cuales se conforma un conjunto de referencias, que son válidas en la medida que garanticen la eficiencia, la eficacia y la efectividad de la organización, convirtiendo en un estilo de vida que favorece el desenvolvimiento institucional.

Los docentes se sienten identificados y comprometidos con la institución para la que trabajan, a pesar de que algunos docentes llevan poco tiempo laborando en la institución, refieren que conocen los objetivos de la institución y conocen los resultados que deben alcanzar en su punto de trabajo, y frecuentemente se establece metas por periodos cortos de tiempo. 
Los docentes manifestaron que muestran en todo momento una tendencia a fortalecer el ambiente de trabajo donde los valores morales y éticos son las vías para alcanzar las metas y objetivos a fin de crear un ambiente estable.

El 22.73\% manifiestan que a veces existe colaboración entre áreas y que el trato para todos los trabajadores es igual. Los docentes manifestaron que casi nunca ante un conflicto institucional reacciona negativamente lo que resulta favorable a la institución.

Tabla 3. Relación entre inteligencia emocional y cultura organizacional de los docentes de la Institución Educativa Privada Andrés Avelino Cáceres, 2015

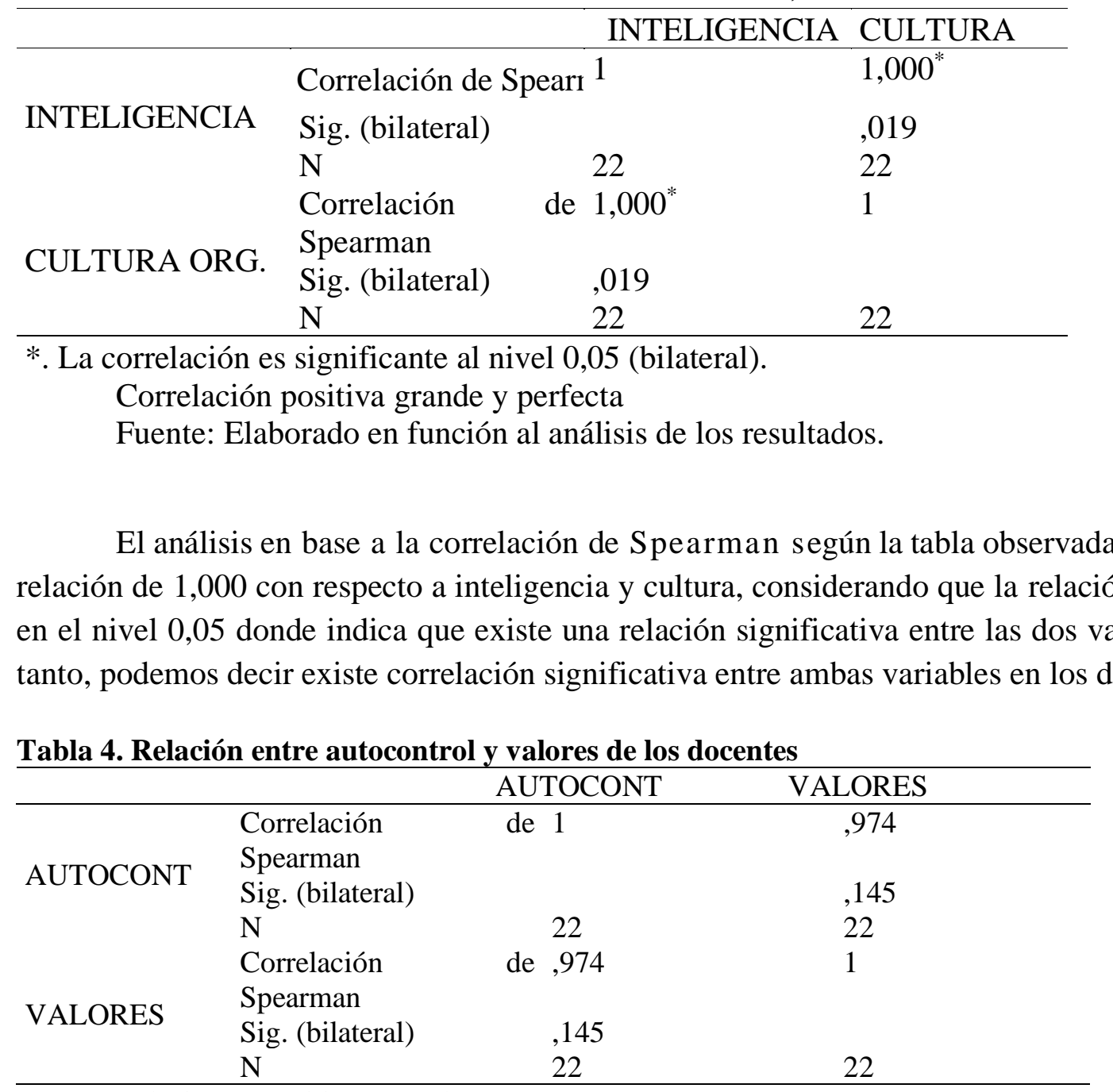

*. La correlación es positiva muy alta

Fuente: Elaborado en función al análisis de resultados.

La relación es directa entre autocontrol y valores, por lo que los docentes predominan el manejo de las emociones durante el trabajo educativo, previendo controlar otras acciones que no corresponden al actuar, asumiendo con responsabilidad y valorando las acciones de los demás, logran controlar sus impulsos emocionales ante cualquier problema o situación conflictiva del trabajo dentro de la organización, y recordando siempre los valores que identifica a cada persona. 
Tabla 5. Relación entre automotivación y objetivos de los docentes de la Institución Educativa Privada Andrés Avelino Cáceres, 2015

\section{AUTOMOTIVACIÓN OBJETIVOS}

\begin{tabular}{llcl}
\hline \multirow{2}{*}{ AUTOMOTIVACIÓN } & Correlación & de 1 & $1,000^{*}$ \\
& Spearman & &, 019 \\
& Sig. (bilateral) & & 22 \\
OBJETIVOS & Correlación & de $1,000^{*}$ & 1 \\
& Spearman & & \\
& Sig. (bilateral) &, 019 & 22 \\
\hline
\end{tabular}

*. La correlación es significante al nivel 0,05 (bilateral).

Correlación positiva grande y perfecta

Fuente: Elaborado en función al análisis de resultados.

En la tabla se obtuvo una relación de 1,000 con respecto a automotivación y objetivos, considerando que la relación significativa en el nivel 0,05. Donde nos indica que existe una relación significativa entre las dos variables.

Tabla 6. Relación entre empatía y actitudes de los docentes de la Institución Educativa Privada Andrés Avelino Cáceres, 2015

\begin{tabular}{llll}
\hline & & EMPATÍA & ACTITUDES \\
\hline \multirow{3}{*}{ EMPATÍA } & Correlación de Spearman & 1 & $1,000^{*}$ \\
& Sig. (bilateral) & &, 019 \\
& $\mathrm{~N}$ & 22 & 22 \\
& Correlación de Spearman & $1,000^{*}$ & 1 \\
\multirow{2}{*}{ ACTITUDES } & Sig. (bilateral) &, 019 & \\
& $\mathrm{~N}$ & 22 & 22 \\
\hline
\end{tabular}

* La correlación es significante al nivel 0,05 (bilateral).

Correlación positiva grande y perfecta

Fuente: Elaborado en función al análisis de resultados.

En la tabla se obtuvo una relación de 1,000 con respecto a empatía y actitudes, considerando que la relación significativa en el nivel 0,05 . Donde nos indica que existe una relación significativa entre las dos variables.

Según los resultados obtienen relación positiva las dimensiones entre empatía y actitudes de los docentes de la IE, por lo que sus actitudes son de agrado al saber escuchar y ponerse en el lugar de los demás, comprendiendo atentamente, hasta llegando a asesorar casos de la vida real en forma concreta. Los docentes en las reuniones, charlas, congresillos se evidencian fundamentalmente la comprensión, consejos vividos, asimismo reflexionan aceptando las críticas constructivas llegando a soluciones. 
Tabla 7. Relación entre habilidades sociales y liderazgo de los docentes de la Institución Educativa Privada Andrés Avelino Cáceres, 2015

HABILIDADES LIDERAZGO

\begin{tabular}{llll}
\hline & Correlación de & 1 &, 235 \\
HABILIDADES & Spearman & & \\
& Sig. (bilateral) & &, 849 \\
& N & 22 & 22 \\
& Correlación de &, 235 & 1 \\
LIDERAZGO & Spearman & & \\
& Sig. (bilateral) &, 849 & \\
& N & 22 & 22 \\
\hline
\end{tabular}

*. La correlación es positiva baja

Fuente: Elaborado en función al análisis de resultados.

Se aprecia que en estas dimensiones no se asemejan a una relación favorable ya que las habilidades y el liderazgo de los docentes evidencian diferentes estilos y formas de llegar a la organización social, es decir un grupo de docentes aplican el estilo tradicional o transaccional, esto quiere decir que los docentes solo intervienen cuando existe algún tipo de recompensa, incentivo o castigo. Mientras que el otro grupo de docentes son más transformacionales, es más dinámico son los que inspiran y motivan a un grupo de docentes y son tomados como modelos a seguir, por lo que la relación entre ambas dimensiones se diferencia. Por tanto, las habilidades sociales de comunicación, habilidades de equipo y liderazgo son presenciadas con los diferentes estilos o maneras de llegar frente a la organización cultural.

\section{DISCUSIÓN}

El abordaje de la educación básica con altos estándares de calidad, implica revertir algunos puntos tradicionalmente construidas, de una educación lineal de enseñanza aprendizaje que aún se mantienen en el Perú, sobre todo en la educación pública, que cuyo efecto genera un contexto tenso y conflictivo tanto para los padres de familia como para los docentes, las emociones especialmente las negativas, influyen significativamente en el desempeño académico de los niños (Tiria, 2015). Es necesario una educación que busque incluir en este proceso a las emociones, teniendo presente que la mayoría de nuestras decisiones, incluyendo las complejas como el aprendizaje, están dominadas por las emociones (Valera, 2018). Un estudiante cuyas emociones sean adecuadamente canalizadas, será un estudiante con alto potencial para el desarrollo académico. Los programas de aprendizaje socio-emocional en el aula deberían recoger y enfatizar, tales como atender los sentimientos, comprender las emociones y usarlas en nuestro razonamiento para solucionar problemas (Fernández \& Montero, 2016), dependerá de una adecuada cultura organizacional, de ahí la importancia de abordar estos dos campos estratégicamente necesarios y compatibles, lo que pretende reflejar la investigación.

\section{Inteligencia emocional}

El estudio de las emociones en las ciencias sociales y en las ciencias de la educación son relativamente nuevas. Tomando los sentimientos y emociones como el centro de interés y objeto de estudio, en un contexto educativo (Alterio \& Pérez, 1999). Lo cual no quita el interés por la 
formación en conocimientos, más bien, complementa un vacío considerado antaño, poco relevante. El estudio de las emociones resulta crucial en una educación de calidad, que conlleve al pleno desarrollo humano y de inclusión. Tal como afirma López y De la Garza (2018) proyecta un fructífero futuro en la investigación, llegando a diversos escenarios generados por la globalización y abierta a infinitas posibilidades de desarrollo en una vasta gama de instituciones no solo académicas.

Hablar, escribir y pensar en las emociones es de interés académico y de la ciencia, la vida afectiva ocupa un espacio creciente en el discurso de la postmodernidad y en estos tiempos de pandemia, se espera que los maestros sean razonables y justos, que manejen consistentemente sus mentes, sentimientos y conductas (Vivas, 2003). En los docentes, el abordaje de la parte afectiva resulta favorable y positiva en el aprendizaje significativo, demostrando tanto en las cuestiones sociales y académicas tendencias de empatía y habilidades sociales, pensando que la responsabilidad de los maestros no es solo enseñar conocimientos, sino contribuir al perfeccionamiento de cada uno de los alumnos (Fernández \& Montero, 2016).

La afectividad de los docentes se observa en las reuniones, talleres, escuela de padres, actividades internas y externas de la Institución Educativa, frente a la comunidad educativa, de ello depende muchas veces el éxito de las actividades, gracias a la acción de dominio de capacidades emocionales que manifiestan, predominantemente la empatía y las habilidades sociales, entendiendo que el grado de inteligencia emocional no está determinado genéticamente (Goleman, 1995), si no es una construcción social a través del tiempo y la experiencia.

Los docentes logran controlar sus impulsos emocionales ante cualquier problema o situación conflictiva, utilizando el diálogo para resolverlos, poseen confianza en uno mismo y consideran que al realizar las actividades laborales y educativas lo realizan de la mejor manera, motivándose a cumplir logros y metas trazadas ya sea en lo personal o para la institución, comprendiéndose de manera tal que las actitudes que el docente asuma pueden contribuir o dificultar el aprendizaje de los estudiantes (J. A. García, 2012).

\section{Cultura organizacional}

La Institución Educativa Andrés Avelino Cáceres de la ciudad de Puno asume un esfuerzo sistemático por definir la categoría de cultura adoptando un enfoque empresarial y organizacional. Schein (1985) parte de la premisa de que las organizaciones tienen culturas propias. En un nivel general, se entiende a la cultura organizacional como aquellas dimensiones inconscientes que afectan el modo en que los miembros de una institución piensan, sienten y actúan. Este conjunto de comportamientos y creencias que conglomeran el aspecto simbólico y subjetivo del ser humano, que en términos de Geertz (2003) configura el entramado de significados socialmente construidos. Lo cual se evidencia en el sistema organizativo de la IE. En la actualidad, según como afirma Llanos (2016) "cada vez son más las organizaciones que buscan un elemento diferenciador, y es la cultura organizacional precisamente la que les proporciona su propia personalidad e identidad" (p. 3).

Juega un papel importante el rol de los líderes de la institución, son ellos quienes inspiran al grupo con sus ideas, transmiten la política institucional, los valores institucionales, los cuales 
llegan a convertirse en definiciones compartidas y validadas por los miembros de la organización. Los docentes se identifican con los valores en especial el valor de la responsabilidad en el trabajo y en las actividades académicas dando lo mejor de sí, otro de los valores que muestran son el compañerismo, transparencia, honestidad y respeto dentro de la institución, como afirma Ansa (2009) los valores organizacionales son los principios que caracterizan el comportamiento de las personas dentro de la organización y constituyen la base en el que se apoya la filosofía de la organización; logrando en el caso de los docentes de la IE, relaciones laborables afectuosas y solidarias. Los docentes participan en reuniones de carácter social con los compañeros de trabajo, lo cual resulta favorable, pues crean espacios de integración, se fortalecen los valores, se forma un mejor ambiente de trabajo en función al logro de los objetivos de la institución.

\section{Relación entre inteligencia emocional y cultura organizacional}

Muchas instituciones concentran su esfuerzo y consideran que el fin de sus acciones es la mejora de ingresos, no entendiendo que esto es una consecuencia. La "poca motivación y el bajo rendimiento obedece a que en la mayoría de los casos el interés de la organización se centra en la productividad y desempeño laboral, restándole importancia a la inteligencia emocional y por ende al autocontrol, la estabilidad, la empatía y el desarrollo personal de los colaboradores" (Cutiba et al., 2018, p. 9). Esto a la larga tiene consecuencias negativas para la organización y en la calidad del servicio que brinda.

En el caso de la Institución Educativa Privada Andrés Avelino Cáceres, la relación entre emociones y cultura organizacional es notable en las expresiones artísticas, las actividades deportivas internas y externas y las actividades de expresión cultural en general. "La inteligencia emocional nace como una nueva manera de entender la inteligencia y una útil herramienta para alcanzar tanto las metas y objetivos personales como aquellos organizacionales" (Momenti, 2015). Las emociones son formas que experimentan dentro de la comunidad educativa y las respuestas emocionales reflejan la cultura organizacional, tal y como se reflejan en los resultados de la investigación. Las emociones son favorables en los docentes en estudio y en la organización, siendo sólidas y confiables, lo cual es una manera de mejorar la calidad educativa. El proceso de introspección personal permitió visualizar valores y principios de vida, que exigen asumir responsabilidades en la búsqueda del mejoramiento personal, que influye a su vez el desarrollo de la organización y de la comunidad educativa en general.

En relación con las variables se observa claramente que se obtiene relación positiva perfecta entre inteligencia emocional y cultura organizacional, esto significa que, en la IE los docentes en estudio muestran afectividad emocional favorable para la organización, fundamentalmente en las creencias, costumbres, actividades internas como en las actividades cotidianas, y actividades de interrelación externas.

\section{Relación entre autocontrol y valores}

Los resultados muestran correlación positiva muy alta entre las dimensiones de autocontrol y valores, por tanto, los docentes ejercen control sobre uno mismo teniendo control de los lados positivos y manejan los lados negativos, mostrando una identidad corporativa, premunidos de "un conjunto de valores, creencias y costumbres que en términos generales les da a sus integrantes el 
sentido de pertenencia e identidad comunitaria" (Hernández et al., 2018). Asimismo, los docentes tienen la capacidad de regular la manifestación de una emoción para dar una respuesta controlada y dirigida hacia donde debe estar, proporcionando una respuesta medida de acuerdo con el evento de manera responsable. "La gestión de valores es una estrategia de liderazgo que busca entender y aplicar conocimientos basados en el diálogo sobre valores" (Buitrago \& Sanabria, 2013).

Por tanto, manejan las emociones lo que facilita las tareas en vez de estorbarlas. "El control de las emociones no es una tarea fácil, lo que debemos hacer es que estas emociones jueguen a nuestro favor; la clave está en utilizarlas de forma inteligente" (Cano \& Zea, 2019). En este sentido, se puede decir que toda cultura es valor, tales como la transparencia, los docentes comunican sus pensamientos, sentimientos y propuestas, sin ocultar nada, sin doble sentido, en forma simple y directa. Otra cualidad fundamental de valores es la responsabilidad y el compañerismo que se establece entre colegas de la IE.

\section{Relación entre automotivación y objetivos}

Los resultados muestran correlación positiva perfecta entre las dimensiones de automotivación y objetivos institucionales, esto significa que los docentes saben automotivarse, lo cual ha facilitado la existencia de tendencias emocionales que conducen hacia la consecución de los objetivos propuestos. Ordenan sus emociones motivadoras al servicio de un objetivo esencial. Siendo diferente el abordaje de cada uno de los docentes "debido a que cada individuo tiene un carácter propio y lo que motiva a uno no tiene por qué ser válido para otro. Lo importante es que cada profesional identifique cuáles de los siguientes comportamientos le serán más útiles para mejorar su automotivación" (Maella, 2015).

Según los resultados, los docentes, muestran un alto grado de compromiso, aliándose a las metas de la organización y están dispuestos a hacer sacrificios para lograr un objetivo general. El "autosacrificio puede generar confianza y aceptación extraordinaria en los seguidores"(Ayoub, 2010), como efectos encuentran una sensación de ser útiles en la misión general, utilizan los valores del grupo para tomar decisiones y clarifican sus alternativas y buscan activamente oportunidades para cumplir con la misión del grupo. Además, son optimistas por lograr el objetivo, pese a los obstáculos, "un empleado motivado para la empresa es un producto o servicio de mejor calidad, llevando a la organización al éxito y a un mejor posicionamiento en el mercado" (Ceron, 2015), van tras la meta, no operan por miedo al fracaso, con la seguridad del respaldo institucional, motivado hacia el éxito.

En ambas dimensiones teórica y práctica vivida por los docentes, se observa relación directa, asimismo se comprueba mediante la correlación de Spearman donde la automotivación se relaciona en función a objetivos. Los docentes son los motivadores de las acciones educativas cumpliendo o tranzándose metas, primordialmente en la formación integral del educando, los compromisos educativos, participación en la escuela de padres, actividades internas y externas. Sabiendo que las organizaciones se enfrentan a un ambiente competitivo y de alta incertidumbre, que les demanda adquirir ciertas competencias para adaptarse mejor a su entorno y tener más capacidad de respuesta frente a las situaciones de crisis (Blanco, 2017). En la IE, la situación de crisis vividas, son oportunamente respondidas. 


\section{Relación entre empatía y actitudes}

La capacidad de ponerse en situación del otro, implica el ejercicio de aproximación al punto de vista y a la lógica del otro, actitud fundamental en el ejercicio del profesorado, eso observamos en los docentes de la IE, que ayudan a los demás a desarrollarse fomentando actitudes favorables, recompensando con aliento las virtudes, los logros y el progreso institucional, considerando como "una formación integral que englobe conocimientos académicos y habilidades socio-afectivas" (Fragoso, 2015). Los profesores asesoran y brindan consejos oportunos, asignan tareas que fortalecen y alientan las habilidades del otro, satisfacen las necesidades del otro, por lo que comprenden las necesidades de los demás docentes, buscan maneras de aumentar la satisfacción, ofrecen buen grado de asistencia y analizan el punto de vista de los demás, actuando como asesores de confianza. Para que el alumno aprenda y desarrolle las habilidades emocionales y afectivas relacionadas con el uso inteligente de sus emociones necesita de un educador emocional (Extremera \& Fernandez, 2005).

Como resultados de investigación tenemos una correlación significativa y perfecta, entre las dimensiones de empatía y actitudes presenciando en los docentes una actitud social, captando los sentimientos, necesidades e intereses ajenos, “(...) Los maestros pueden y deben crear el escenario para que el estudiante acierte, y que en los intentos fallidos, aprenda y recree la necesidad de pensar de manera distinta" (Guerrero et al., 2013, p. 374); por tanto, obtienen la predisposición para admitir otras posibilidades, escuchar con concentración o escucha activa y son capaces de comprender pensamientos y sentimientos que no se expresan verbalmente.

\section{Relación entre habilidades sociales y liderazgo}

La relación entre las dimensiones de habilidades sociales y liderazgo es baja según los resultados obtenidos mediante la correlación de Spearman, en el campo conductual: como como saber escuchar, saber pedir colaboración y en el campo emocional: pedir aclaraciones, saber quejarse, reaccionar a la presión del grupo, etc., otras habilidades alternativas a la agresión: responder a una burla, solucionar conflictos (Tapia \& Cubo, 2017), son variadas, los procesos de motivación y convencimiento a los demás según sus diferentes habilidades sociales, según manifiestan los docentes, son espacios que aún faltan consolidarlos.

El liderazgo es la otra cara de la cultura organizacional y para ser un buen líder es necesario desarrollar habilidades sociales, debiendo considerarse que en la gestión educativa no se trata de gestionar aspectos aislados sino, tener en cuenta que entre los diferentes componentes del sistema escolar se dan múltiples interrelaciones que es necesario comprender para mejorar (Martínez, 2010), es decir, el liderazgo organizacional debe considerar el entendimiento global de IE, hacer que sea parte del desarrollo de una cultura organizacional. Como refiere Begoña (2009) un líder "genera un clima de entusiasmo y flexibilidad en el que las personas se sienten estimuladas para ser más creativos y dar lo mejor de sí mismos..., alentar a la cooperación y confianza que solo es posible mediante la inteligencia emocional" (p. 6). En los resultados de investigación este aspecto aún requiere fortalecer. Como afirma García (2017) en futuros proyectos es importante contemplar la implicación de toda la comunidad educativa si realmente se persigue la implantación de la educación emocional a nivel institucional con capacidad de cultivar relaciones, reconocer 
conflictos y solucionarlos, encontrando el tono adecuado para percibir el estado de ánimo de las otras personas.

\section{CONCLUSIONES}

En la educación actual las instituciones educativas, en su cultura organizacional, requieren cambios cualitativamente significativos, entendiendo el servicio como aquella que considere el centro de atención la calidad humana, con docentes emocionalmente inteligentes, capaces de construir empatías y convergencias de las emociones de los miembros de la comunidad educativa, principalmente estudiantes. Docentes que puedan percibir, comprender y regular las emociones propias y de las personas con quienes interactúa para canalizar situaciones estresantes y dar respuestas emocionalmente inteligentes.

Substancialmente, si no se cuenta con una adecuada cultura organizacional, los buenos docentes que cuentan con una inteligencia emocional caerán en el aletargamiento, absorbido por el modelo imperante, contribuyendo al círculo vicioso de una mala calidad educativa.

Estadísticamente, se comprueba mediante la correlación entre la inteligencia emocional y la cultura organizacional, existiendo relación significativa y positiva de los docentes de la institución educativa de la referencia, mostrando afectividad emocional favorable en la organización. Son notables en las acciones y expresiones, por tanto, la inteligencia emocional, mediante la identificación y manejo de emociones, le permite al docente mantener una percepción de la organización lo que se traduce en la delimitación de la cultura organizacional en el cual interactúa, definiendo así aptitudes, actitudes y reacciones en orden a la percepción que tienen y asumen dentro de la organización.

\section{REFERENCIAS BIBLIOGRÁFICAS}

Alterio, G., \& Pérez, H. (1999). Inteligencia emocional: Teoría y praxis en educación. Revista Iberoamericana de Educación, 1-5.

Ansa P, M. (2009). Valores institucionales en el personal administrativo de la Universidad del Zulia. Formación Gerencial, 8(2), 256-271.

Ayoub, J. L. (2010). Estilos de liderazgo y sus efectos en el desempeño de la administración pública mexicana. Universidad Autónoma de Madrid.

Begoña, L. de D. (2009). Dirigir y educar con inteligencia emocional. Ponencia del VII congreso de Educación y Gestión, 1-10.

Blanco, L. (2017). La inteligencia emocional como elemento que contribuye al mejoramiento del clima organizacional. El caso de Asoingeniería LTDA. Pontificia Universidad Javeriana.

Buey, M. L. (2002). Importancia de la inteligencia emocional: un nuevo reto para la orientación educativa. Educación XXI, 5. https://doi.org/10.5944/educxx1.5.1.384

Buitrago, M. del P., \& Sanabria, J. E. (2013). La cultura organizacional en las instituciones educativas a través de la evaluación de su sistema de comunicación interna. Universidad Pedagógica Nacional. 
Cano, S. R., \& Zea, M. (2019). Manejar las emociones, factor importante en el mejoramiento de la calidad de vida. Revista Logos, Ciencia \& Tecnología, 12(1). https://doi.org/10.22335/rlct.v4i1.169

Cebriá, N. (2017). Educación Emocional, evolución del concepto e implicaciones en el marco legal de la escuela Primaria.

Ceron, J. (2015). La importancia de la motivación en las empresas. En Repositorio de la Universidad Militar Nueva Granada.

Cutiba, K., García, Z., \& Laguna, L. (2018). Inteligencia emocional y su influencia en la cultura organizacional.

Diaz, B. (2017). La escuela tradicional y la escuela nueva: "análisis desde la pedagogía crítica”. Universidad Padagógica Nacional.

Extremera, N., \& Fernandez, P. (2005). La Importancia de desarrollar la inteligencia emocional en el profesorado. Revista Iberoamericana de Educación, 1681-5653.

Extremera, N., \& Fernández, P. (2003). La inteligencia emocional en el contexto educativo: hallazgos científicos de sus efectos en el aula. Revista de educación, 332, 97-116.

Fernández, A. M., \& Montero, I. (2016). Aportes para la educación de la inteligencia emocional desde la educación infantil. Revista Latinoamericana de Ciencias Sociales, Niñez y Juventud, 14, 53-66. https://doi.org/10.11600/1692715x.1412120415

Fragoso, R. (2015). Inteligencia emocional y competencias emocionales en educación superior, ¿un mismo concepto? Revista Iberoamericana de Educacion Superior, 6(16), 110-125. https://doi.org/10.1016/j.rides.2015.02.001

García, E. (2017). Formación del profesorado en educación emocional: Diseño, aplicación y evaluación. En Programa de Doctorado: Educación y Sociedad. Universitat de Barcelona.

García, J. A. (2012). La educación emocional, su importancia en el proceso de aprendizaje. Revista Educacion, 36(1), 1-24.

Geertz, C. (2003). La interpretación de las culturas (XX-Edito).

Goleman, D. (1995). Inteligencia Emocional (Kairós).

Guerrero, J. I., Castillo, E. J. S., Chamorro, H. G., \& Isaza, G. (2013). El error como oportunidad de aprendizaje desde la diversidad en las prácticas evaluativas. Plumilla Educativa, 12(2), 361-381. https://doi.org/10.30554/plumillaedu.12.388.2013

Hernández, J. E., Antonieta, J., Escalera, L., Castillo, S. P., \& Morales, H. (2018). Acción colectiva, cultura organizacional y desarrollo comunitario en el contexto educativo y de bienestar del grupo menonita en Cuauhtémoc, Chihuahua, México / Collective Action, Cultural Organization and Community Development in the Context of Education. RIDE Revista Iberoamericana para la Investigación y el Desarrollo Educativo, 9(17), 798-836. https://doi.org/10.23913/ride.v9i17.406

Llanos, M. (2016). La cultura organizacional eje de acción de la gestion humana. En Universidad Ecotec. https://doi.org/10.1017/CBO9781107415324.004 
López, J. A., \& De la Garza, M. T. (2018). Tecnología y aprendizaje organizacional factores influyentes sobre la satisfacción profesional en residentes de pregrado. Actualidades Investigativas en Educación, 18(2), 1-23. https://doi.org/10.15517/aie.v18i2.33167

Maella, P. (2015). Las claves de la automotivaciòn en el trabajo. IESE Business SchoolUniversidad de Navarra, 3, 1-13.

Martínez, C. (2010). Gestión de directores y cultura organizacional en instituciones educativas de secundaria - Red $N^{\circ} 7$ del Callao. Universidad San Ignacio de Loyola.

Momenti, V. (2015). Inteligencia emocional y cultura organizacional. Su rol, desarrollo y relacion en el sector bancario argentino. Universidad Torcuato Di Tella.

Olmos, M., \& Socha, K. (2006). Diseño y validación mediante jueces expertos del instrumento para evaluar cultura organizacional. En Universidad de la Sabana. Universidad de la Sabana.

Schein, E. (1985). La cultura organizacional y el liderazgo. Una visión dinámica (Plaza y Ja).

Tapia, C. P., \& Cubo, S. (2017). Habilidades sociales relevantes: percepciones de múltiples actores educativos. Magis, 9(19), 133-148. https://doi.org/10.11144/Javeriana.m9-19.hsrp

Tiria, D. J. (2015). La educación emocional dentro de los procesos de enseñanza aprendizaje de los niños y niñas de 4 y 5 primaria. En Tecnológico de Monterrey. Colombia. Tecnológico de Monterrey.

Valenzuela, A. del C., \& Portillo, S. A. (2018). La inteligencia emocional en educación primaria y su relación con el rendimiento académico. Revista Electrónica Educare, 22(3), 1-15. https://doi.org/10.15359/ree.22-3.11

Valera, O. (2018). La inteligencia emocional y el aprendizaje eficiente de los alumnos del programa de maestría sede - Huancayo. Universida Nacional de Educación Enrique Guzmán y Vallle.

Vivas, M. (2003). La educación emocional: conceptos fundamentales. Sapiens. Revista Universitaria de Investigación, 4(2), 22. https://doi.org/10.1016/j.jinf.2012.02.014

Weisinger, H. (1998). La Inteligencia Emocional en el trabajo. Editorial Javier Vergara. 